\title{
2020 Reviewers
}

Ádám, Norbert Adamne Major, Andrea

Agbinya, Johnson

Andoga, Rudolf

Arahal, Manuel R.

Atanasova - Pachemska, Tatjana

Baklanov, Alexander

Bakucz, Peter

Barányi István

Bednar, Peter

Belina, Károly

Berke, Szilárd

Bobrov, Leonod

Bonilla, Javier

Choroś, Kazimierz

Csobán, Attila

Czakó, Bence Géza

Czifra, Árpád

de la Calle, Alberto

Dömötör, Ferenc

Drexler, Dániel András

Erdélyi, Viktor

Fábián, Enikő Réka

Főző, Ladislav

Fülöp, Zoltan

Garai-Fodor, Monika

Gašpar, Vladimír

Gonda, Viktor

Grion, Valentina

Gyarmati, Gábor

Györök, György

Hargitai, Hajnalka

Horecky, Jan

Horváth, Richárd

Hrabovsky, Jan
Ispány, Márton

Jelacic, Denis

Jukic, Samed

Juříková, Martina

Karpov, Alexey

Kasanicky, Tomas

Katona, Attila

Katona, Jozsef

Kaya, Heysem

Kopacek, Peter

Kovács, László

Kucharcikova, Alzbeta

Laššák, Miroslav

Lipovský, Pavol

Lucka, Maria

Machová, Kristína

Magyar, Attila

Mankovits, Tamás

Matis, Martin

Mikó, Balázs

Molnár, György

Myskova, Renata

Nagy, Dénes Ákos

Pál Ex Apró, Magdolna

Paniti, Imre

Papcun, Peter

Paralic, Jan

Pawel Dobrzanski

Pedro, Rodriguez

Pokorádi, László

Polishchuk, Volodymyr

Precup, Radu-Emil

Prochazka, Ales

Reicher, Regina Zsuzsánna

Rövid, András

Schreiner, Michal 
Schrötter, Martin

Simandi, Szilvia

Smieszek, Miroslaw

Stachová, Katarína

Steingartner, William

Svec, Marek

Szabó, Attila

Szénási, Sándor

Takács, Árpád

Tar, József

Toth, Janos

Tóth, János

Vaispacher, Tomas

Vajdova, Iveta

Vámossy, Zoltán

Vascak, Jan

Venkatraman, Praburaj

Woda, Marek

Wójcik, Waldmar

Závadský, Jan

Zsidai, László 